\title{
Necrotizing fasciitis following transobturator tape procedure: a case report and literature review
}

\author{
Kwang Yeom Lee, MD; Jae Ang Sim, MD, PhD; Sheen Woo Lee, MD, PhD; ${ }_{;}^{*}$ Tae Beom Kim, MD;* \\ San Jin Yoon, MD, PhD; Kyung Seo Park, MD; ${ }^{*}$ Khae-Hawn Kim, MD, PhD*
}

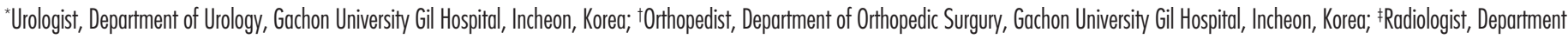
of Diagnostic Radiology, Gachon University Gil Hospital, Incheon, Korea

Cite as: Can Urol Assoc J 2011;5(4):e65-e68; D01:10.5489/cuai.10104

\section{Abstract}

A 51-year-old diabetic woman was referred to our unit with a history of increasing pain in her right thigh and gait disturbance, 10 days following surgery for treatment of stress urinary incontinence with a transobturator tape. Examination elicited a tender right thigh associated with swelling and erythema. Inspection of the vagina revealed an exposed mesh through the vaginal erosion. Plain radiographs and magnetic resonance imaging revealed large thigh abscesses suggestive of gas gangrene from the pelvis to the calf. The mesh was completely removed; extensile incisions, as well as aggressive debridement of all necrotic tissue in the thigh and calf, were performed. A diagnosis of necrotizing fasciitis with gas extending to the lower leg level was confirmed. After mesh removal, abscess drainage and debridement, the patient recovered with antibiotics and daily wound care. Three months after the operation, the patient showed no recurrence of the infection.

\section{Introduction}

The tension-free vaginal tape (TVT) procedure has long been considered the gold standard to treat female stress incontinence. Since the introduction of TVT in 1995, other tapes and minimally invasive treatments have arisen. The transobturator tape (TOT) procedure was first described in 2001 as an alternative to traditional TVT procedures; TOT decreases the risk of bladder, digestive and vascular injuries associated with the retropubic route. ${ }^{1}$ The TOT procedure entails the placement of the polypropylene mesh under the mid-urethra as the tunneler is passed through the obturator foramina. As such, the potential to damage the contents of the obturator foramen, formation of adductor compartment abscess and transient inner thigh pain are associated with the TOT approach. We report a case of a necrotizing fasciitis in the leg manifesting 10 days following a TOT procedure.

\section{Case report}

A 51-year-old woman on oral hypoglycemics for type II diabetes mellitus was referred to our unit with a history of increasing pain in her right thigh and gait disturbance. She had undergone a TOT procedure 10 days previously for stress urinary incontinence at a gynecologic hospital. On examination, she was afebrile and hemodynamically stable. Her right medial thigh was tender, swollen and erythematous. Inspection of the vagina revealed vaginal erosion and discharge from the right anterior vaginal wall, and the mesh was exposed through the vaginal erosion. Moderate tenderness was elicited on the right vaginal wall.

Urinalysis was unremarkable and the laboratory investigations revealed the following outcomes: elevated white blood count (23 $600 \mathrm{cc})$, C-reactive protein $(4.43 \mathrm{mg} / \mathrm{dL})$, blood sugar (362 mg/dL) and $\mathrm{Hb}$ A1C level (10.5\%). Plain radiographs of the pelvis and legs showed extensive emphysema with air fluid levels from the pelvis to the calf, suggestive of gas gangrene (Fig. 1). Magnetic resonance imaging (MRI) revealed large abscesses (larger than $4 \mathrm{~cm}$ ) in the pelvis and thighs on the right side (Fig. 2).

Based on these findings, the decision to perform an emergency operation was made. Under spinal anesthesia, urethro-cystoscopy was normal but the mesh was migrated to the proximal urethra. Subsequently, the mesh was removed completely through a $2-0 \mathrm{~cm}$ midline incision in the anterior vaginal wall. After removing the mesh, the endopelvic fascia was incised and the thigh abscesses were penetrated through the incision site. Subsequently, 400 cc of yellowwhitish pus with offensive odor was drained. To manage the thigh abscess, an orthopedic surgeon performed extensile incision, drainage and aggressive debridement, including the liquefying fascia. About $500 \mathrm{cc}$ of foul smelling reddish pus was released. Additional incisions of the knee and the calf were performed to manage emphysema extending to the lower leg level. A diagnosis of necrotizing fasciitis with gas formed subcutaneously and beneath the fascia of the muscles extending to the lower leg was confirmed. The 


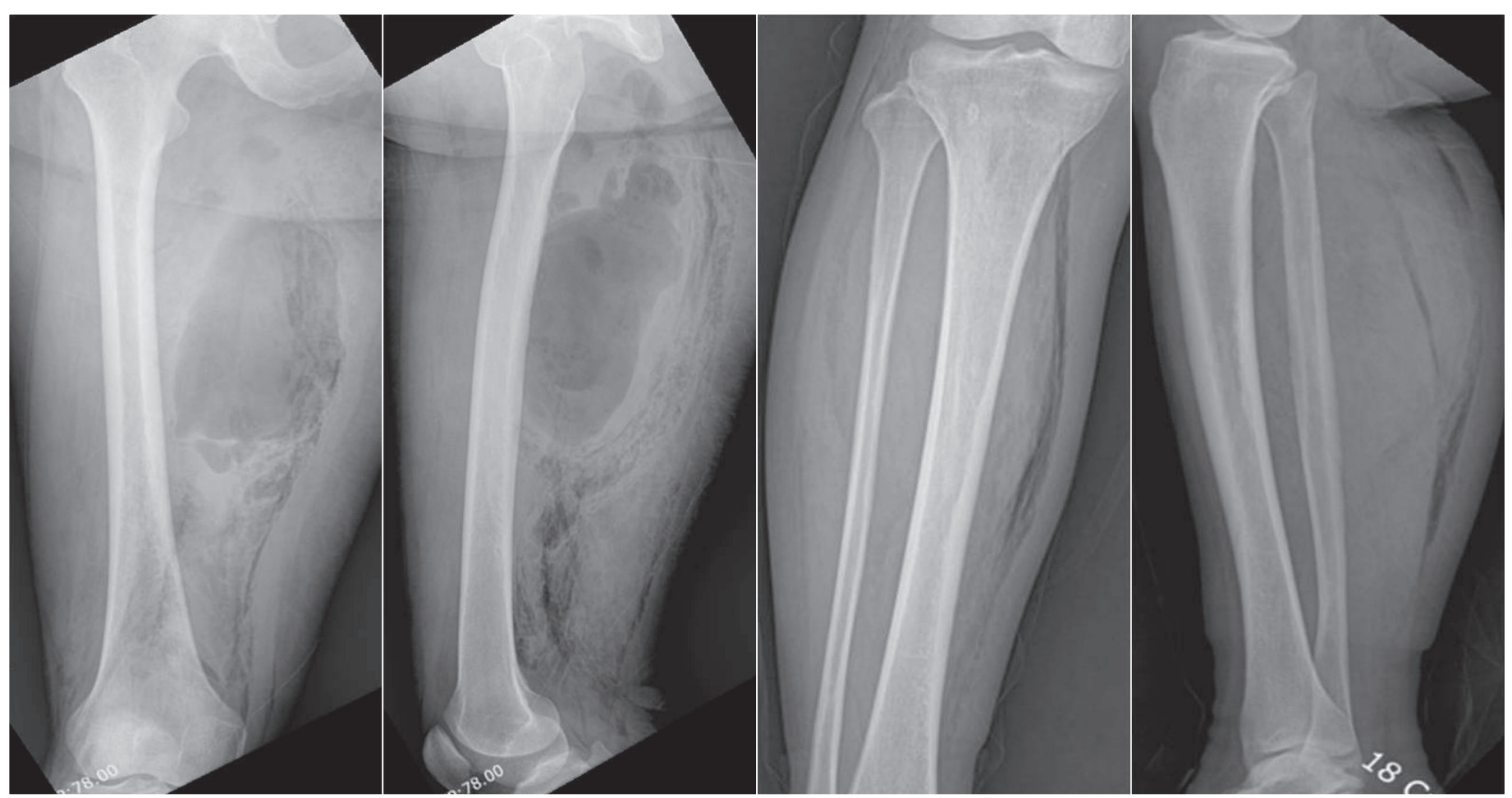

Fig. 1. Plain radiographs of right leg. Right femur anteroposterior (A) and lateral (B) radiographs show extensive soft tissue emphysema in the right posteromedial thigh and lower pelvic area, with deep-seated air-filled cystic lesion suggestive of abscess formation. The radiographs of lower leg (C, D) show the soft tissue emphysema extending inferiorly to lower calf.

incisions on the lower extremity and vaginal wound were left open with drains and packing in place, and the patient received daily repeat inspection and debridement.

After the mesh removal and abscess drainage, pus culture from the wound revealed Streptococcus anginosus, sensitive to ampicillin, cefotaxime, penicillin, clindamycin and vancomycin. The patient's wound was covered with antibiotics therapy (piperacillin + tazobactam, ceftriaxone) and daily betadin-soaked dressing in the operating theatre. A repeat wound culture, after initiating antibiotics revealed no bacterial growth and the wound, was improved. Four weeks after the operation, a secondary repair was performed in the thigh and the calf, but an anterior vaginal repair was not performed due to spontaneous healing. Three months following mesh removal and abscess drainage, the patient had no evidence of infection and returned to daily activities.

\section{Discussion}

The TOT procedure is a minimally invasive surgical procedure to treat stress urinary incontinence; it bypasses the retropubic approach and, ultimately, avoids the bladder, the gastrointestinal tract and other major vessels. ${ }^{1}$ However, due to the anatomical route of the TOT along the obturator fossa, there have been reports of thigh and obturator abscess following the procedure. ${ }^{2-10}$ However, a minority of patients experienced severe infectious complications, as with our patient. In most cases, such serious complications were due to vaginal erosion of the tape and subsequent infections, ${ }^{2-10}$ and the site of infection (obturator or thigh) was explained by the course through the anatomical structures traversed by the tunneler. Among the muscles traversed, the adductor brevis is the first, followed by obturator externus and then internus. ${ }^{7}$ The mechanism of infection in our patient was likely due to vaginal erosion.

Clinically, vaginal erosion may present with vaginal pain, discharge and bleeding, dyspareunia, dysuria and recurrent urinary infections. ${ }^{11}$ However, it may remain asymptomatic or noticed by the patient's partner during intercourse.

The true mechanism of action of tape erosion is poorly understood and there are probably multiple factors, which include tape lying too superficial (especially in the lateral vaginal sulcus), inadequate vaginal incision suturing, wound infection, impaired wound healing and foreign body rejection. ${ }^{11}$ Another important issue to address is the type of tape used. Birch illustrated that although porosity, interstice size and stiffness of the mesh material are important, it is the interaction at the tissue interface which is ultimately important, especially when you consider the potential complications of mesh erosion and extrusion. The ideal prosthesis should elicit minimal initial inflammation and cellular response, followed by vascular and fibroblastic infiltration. ${ }^{12}$ In our 


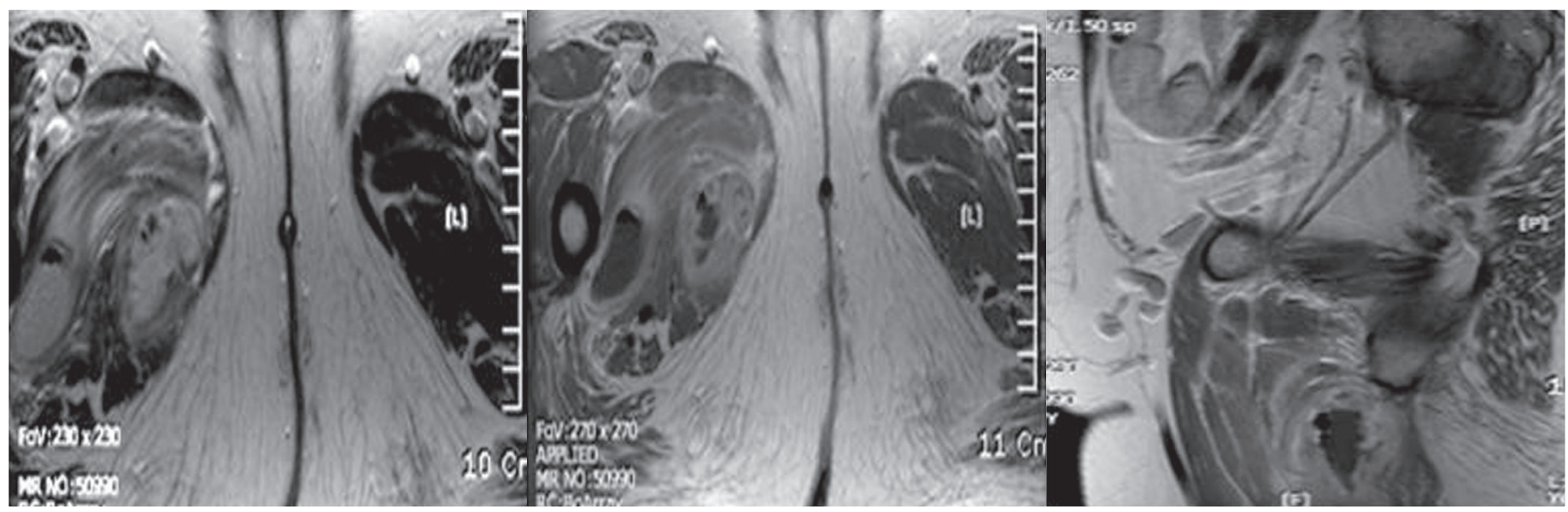

Fig. 2. Magnetic resonance imaging of pelvis. Axial T2-weighted (A) and contrast-enhanced T1-weighted (B) images show myositis containing thick-walled cystic lesions in the right thigh muscle. Sagittal contrast-enhanced T1-weighted image (C) show air-fluid level in the cystic lesion suggestive of abscess cavity probably associated with air-forming bacterial infection.

case, the possible causes of erosion and infection could be due to diabetes mellitus, which renders the patient at a higher risk of infection. However, we could not identify any surgical error or fault in the mesh material (monofilament polypropylene) used.

In this case, the vagina was the likely the source of infection. This finding is supported by the fact that anaerobic Streptococcus anginosus (S. milleri group), which is commonly found in the mouth, throat, gastrointenstinal tract and vagina, was cultured from the abscess supports this. ${ }^{13}$ The S. milleri bacterial group produces toxins, inflammation and toxic shock-like syndrome similar to group A beta-streptococcus, and appears to be a pathogen that causes abscess and serious complications, such as necrotizing fasciitis and sepsis accompanied by disseminated intravascular coagulation..$^{6,14}$ In other case reports, Chlamydia, ${ }^{3}$ streptococcus viridians, ${ }^{4}$ Peptostreptococcus, ${ }^{5,9}$ Bacteroides fragilis, Fusobacterium nucleatum, Staphylococus epidermidis, Streptococcus intermedius, Streptococcus agalactiae and Candida albicans ${ }^{9}$ were cultured from the abscess in the thigh and obturator, suggestive of a vaginal source of infection.

Necrotizing fasciitis is a severe form of soft tissue infection that primarily involves the superficial fascia. It may be due to insults to the integumentary system, or from hematogenous spread. It may spread rapidly to involve the whole limb. For these reasons early debridement and antibiotic therapy are essential to avoid a fatal outcome. Most patients have some form of chronic debilitating diseases, such as diabetes mellitus, alcohol abuse and renal insufficiency. Of these conditions, diabetes mellitus was the most common, present in $57 \%$ of patients. ${ }^{15}$

The clinical presentation entails progressive skin changes. Early on, only tenderness, swelling, erythema and warm skin are present. In addition to common presenting symptoms, such as swelling and redness, patients have severe pain, high fever, hypotension and other signs of systemic toxicity.
The diagnosis depends on recognition of the characteristic rapidly progressive clinical course. In addition, during wound exploration, loss of fascial integrity along the tissue planes and frank evidence of muscle involvement are also diagnostic. Imaging studies, such as computed tomography (CT) or MRI, cannot visualize necrotic tissues, but they will often show extension of inflammation, abscesses and gas. Our patient had severe infection of the deeper tissues on her right thigh and rapid spread along the fascia to the lower leg within days after the TOT procedure. Plain radiographs showed extensive emphysema from the pelvis to the calf, with air fluid level in the right thigh, suggestive of thigh abscess and gas gangrene. An MRI was performed subsequently and it was useful in rapidly establishing the diagnosis of necrotizing fasciitis with thigh abscess, and its use should be considered in patients with apparent signs of tissue infection or thigh abscess after placement of a TOT. ${ }^{8}$

Flam and colleagues reported that immediate debridement with wide incision and excision of all necrotic tissue and extensive fasciotomy where indicated, with complete removal of the tape are essential, followed by vigilant surgical re-examination of the infected area. ${ }^{6}$ In addition, the authors recommended broad-spectrum antibiotics and hyperbaric oxygen therapy (a "high dose oxygen inhalation and diffusion therapy") to manage severe necrotizing infections with rapid progression and systemic toxemia. Our patient improved with immediate muscle debridement and fasciotomy; the wound was covered with broad-spectrum antibiotics and dressed daily with betadin without hyperbaric oxygen therapy.

\section{Conclusion}

All patients who undergo the TOT procedure should be informed of the risks of erosion and infection. In addition, they should be educated to be vigilant about pain and vagi- 
Lee et al.

nal discharge, which may be the first signs of severe infectious complications.

\section{Competing interests: None declared.}

This paper has been peer-reviewed.

\section{References}

1. Delorme E. Transobturator urethral suspension: mini-invasive procedure in the treatment of stress urinary incontinence in women [article in French]. Prog Urol 2001;11:1306-13.

2. Araco $F$, Gravante $G, D E$ Vita $D$, et al. Obturator abscess with spread to the thigh after three years from a transobturator procedure. Aust N Z J Obstet Gynaecol 2009;49:335-6.

3. Zumbé J, Porres D, Degiorgis PL, et al. Obturator and thigh abscess after transobturator tape implantation for stress urinary incontinence. Urol Int 2008;81:483-5.

4. Karsenty G, Boman J, Elzayat E, et al. Severe soft tissue infection of the thigh after vaginal erosion of transobturator tape for stress urinary incontinence. Int Urogynecol J Pelvic Floor Dysfunct 2007; 18:207-12.

5. Goldman HB. Large thigh abscess after placement of synthetic transobturator sling. Int Urogynecol J Pelvic Floor Dysfunct 2006; 17:295-6.

6. Flam $F$, Boiisen $M$, Lind $F$. Necrotizing fasciitis following transobturator tape treated by extensive surgery and hyperbaric oxygen. Int Urogynecol J Pelvic Floor Dysfunct 2009;20:113-5.
7. DeSouza R, Shapiro A, Westney OL. Adductor brevis myositis following transobturator tape procedure: a case report and review of the literature. Int Urogynecol J Pelvic Floor Dysfunct 2007;18:817-20.

8. Deffieux X, Donnadieu AC, Mordefroid M, et al. Prepubic and thigh abscess after successive placement of two suburethral slings. Int Urogynecol J Pelvic Floor Dysfunct 2007: 18:571-4.

9. Benassi G, Marconi L, Accorsi F, et al. Abscess formation at the ischiorectal fossa 7 months after the application of a synthetic transobturator sling for stress urinary incontinence in a type II diabetic woman. Int Urogynecol J Pelvic Floor Dysfunct 2007; 18:697-9.

10. Rardin CR, Moore R, Ward RM, et al. Recurrent thigh abscess with necrotizing fasciitis from a retained transobturator sling segment. J Minim Invasive Gynecol 2009; 16:84-7.

11. Tsivian A, Kessler 0 , Mogutin B, et al. Tape related complications of the tension-free vaginal tape procedure. I Urol 2004;171:762-4.

12. Birch C. The use of prosthetics in pelvic reconstructive surgery. Best Pract Res Clin Obstet Gynaecol 2005;19:979-91.

13. Gossling J. Occurrence and pathogenicity of the Streptococcus milleri group. Rev Infect Dis 1988;10:25785.

14. Fuijyoshi T, Okasaka T, Yoshida M, et al. Clinical and bacteriological significance of the Streptococcus milleri group in deep neck abscesses [article in Japanese]. Nippon Jibiinkoka Gakkai Kaiho 2001;104:147-56.

15. Hung $\mathrm{CC}$, Chang SC, Lin SF, et al. Clinical manifestations, microbiology and prognosis of 42 patients with necrotizing fasciitis. J Formos Med Assoc 1996;95:917-22.

Correspondence: Dr. Khae-Hawn Kim, Department of Urology, Gachon University Gil Hospital, 1198, Guwol-dong, Namdong-gu, Incheon 405-760, Korea; fax: +82-32-460-3840; kimch099@ gilhospital.com 